\title{
Internacionalización de la empresa familiar: un modelo prospectivo
}

\author{
Jenny Romero-Borre ${ }^{{ }^{*}}$, Lissette Hernández-Fernández ${ }^{2,3}$, Rafael Portillo-Medina ${ }^{2}$, Ana Hernández-Chacín ${ }^{4}$ \\ (1) Universidad de la Costa, Facultad de Ciencias Económicas, Barranquilla-Colombia. (correo-e: jromero58@cuc.edu.co) \\ (2) Facultad de Ciencias Económicas, Programa de Economía, Universidad del Atlántico, Barranquilla - Colombia \\ (correo-e:_lisettehernandez@mail.uniatlantico.edu.co; rafaelportillo@mail.uniatlantico.edu.co) \\ (3) Facultad de Ciencias Económicas, Administrativas y Contables, Maestría en Administración de Empresas - MBA, \\ Universidad de Santander, Bucaramanga, Colombia. \\ (4) Postgrado e Investigación, Universidad de Yacambú, Barquisimeto, Venezuela (correo-e: \\ hernandezanaemilia@gmail.com)
}

* Autor a quien debe ser dirigida la correspondencia.

Recibido Jun. 9, 2021; Aceptado Ago. 17, 2021; Versión final Sept. 15, 2021, Publicado Feb. 2022

\section{Resumen}

El principal propósito de esta investigación es definir un modelo prospectivo para explicar la dinámica de la internacionalización de empresas familiares venezolanas. La metodología utilizada transita distintas fases investigativas, comenzando por la descriptiva, construida a partir de los referenciales teóricos y empíricos, cuyo procesamiento arroja un sistema de variables. Luego, se emplea el análisis estructural, cuya técnica requiere la aplicación del programa computarizado MicMac para obtener el grado de motricidad y dependencia de las variables, identificando aquellas que tuvieran la mayor fuerza explicativa a partir de la cual se establece el modelo. Los resultados muestran que las empresas requerirán de recursos y capacidades necesarios para fortalecer su competitividad internacional. Los empresarios son conscientes que para darle continuidad al negocio es necesaria la transmisión efectiva a los miembros de la siguiente generación de los valores y principios que constituyen la iniciativa empresarial. Se concluye que los empresarios valoran, como un factor relevante, el preservar la propiedad en exclusiva para la familia.

Palabras clave: empresa familiar; internacionalización; prospectiva; modelo; competitividad

\section{Internationalization of family businesses: a prospective model}

\begin{abstract}
The main objective of this research study is to define a prospective model that explains the dynamics of the internationalization of Venezuelan family businesses. The methodology applied undergoes different investigative phases, starting with a descriptive phase built from theoretical and empirical references, which resulted in system variables. To establish the model, a structural analysis is performed on MicMac software to obtain the variables' degree of motor skills and dependences and to identify the variables with the greatest explanatory power. The results show that companies require resources and capacities to strengthen their international competitiveness. Entrepreneurs are aware that to provide continuity to their businesses it is necessary to effectively pass on the values and principles that constitute a business to the next generation. It is concluded that entrepreneurs place significant value on maintaining their business properties exclusively within their families.
\end{abstract}

Keywords: family business; internationalization; prospective; model; competitiveness 


\section{INTRODUCCIÓN}

La empresa familiar logra conjugar dos sistemas sociales: la familia, cuya esencia es el amor y la compresión entre sus miembros, sobre el cual se edifican los valores del individuo y de la sociedad (Corral y Rébori, 2019). La empresa, por su parte, está orientada básicamente a la lógica del mercado, que se fundamenta en los postulados de la economía neoclásica, que asume que el empresario es un agente económico racional, encauzado hacia la maximización del beneficio económico y a la acumulación de capital, entre otras finalidades (Romero et al., 2015). La articulación que se da entre estas dos dinámicas hace que estas organizaciones tengan unas características propias y unos objetivos, que en algunas ocasiones son contradictorios, haciéndolas propensas al riesgo y a la desaparición de ambos sistemas (familia y empresa). Dichas contradicciones surgen por el hecho que algunas decisiones priorizan el logro de objetivos no económicos, es decir, de naturaleza afectiva, comprometiendo su desempeño o competitividad en el mercado (Kellermanns et al., 2012; Cabrera-Suárez et al., 2014).

Al respecto, algunos investigadores señalan que tales contradicciones surgen por el deseo de la familia de mantener el control de la empresa, preservar lazos y valores familiares, así como el prestigio y la imagen de la empresa (Tsang, 2002; Berrone et al., 2012). No obstante, esta actitud ha llevado al fracaso a numerosas empresas, pues aproximadamente el $70 \%$ de estas unidades no consigue llegar a la segunda generación, mientras el $90 \%$ no alcanza la tercera (Hernández y Portillo, 2016). Incidiendo, además, la mala planificación de la sucesión, los conflictos familiares, las diferentes visiones entre generaciones y la falta de profesionalización. Ante estos problemas que puedan presentar, son empresas que cada día cobran mayor preeminencia, por su contribución especial en la producción, empleo e ingreso, siendo pilares fundamentales en la economía y aún en situaciones difíciles, siguen en pie dispuestas a regenerar el tejido empresarial. En consecuencia, se resalta su importancia en el ámbito económico y social de acuerdo con Petrủ et al. (2019), requiriendo el apoyo y la promoción de organismos de carácter público y privado, nacional e internacional, para la creación y permanencia en el tiempo.

La internacionalización constituye una vía para crecer y fortalecerse productivamente, dado que amplia los modos de operación e incrementa los ingresos al tener una base de clientes extranjeros (Kampouri et al., 2017; Metsola et al., 2020; Niittymies y Pajunen, 2020). Cabe destacar, que los países desarrollados y exportadores apoyan las medidas de internacionalización de pequeñas y medianas empresas, ante su contribución significativa a PIB (Augusto-Felício et al., 2016; Petrů et al., 2019). Sin embargo, diversas teorías referencian el temor que sienten los empresarios familiares para asumir los retos de la incursión al mercado internacional, pues consideran que el patrimonio familiar se ve amenazado debido a que dicho proceso exige la inversión de grandes recursos, por esta razón suelen retrasar la toma de esta decisión, conformándose con abarcar únicamente el mercado local (Pukall y Calabrò, 2014; Clark et al., 2018).

Este elevado riesgo en el emprendimiento de negocios en un contexto ajeno a su espacio productivo, evidencia parte de la relación negativa entre propiedad familiar e internacionalización, que se expresa en la propensión del sector en retrasar la incursión en mercados internacionales o simplemente conformarse con abarcar sólo el mercado local (Boellis et al., 2016), situándose el componente afectivo propio de estas empresas como un elemento perturbador de la racionalidad del negocio, específicamente en el ámbito internacional. Bajo estas consideraciones, este artículo se orienta a la generación de un modelo de tipo prospectivo para explicar la dinámica de la internacionalización en las empresas familiares venezolanas. La muestra objeto de estudio se definió tomando en cuenta la información aportada por el Ministerio de Industrias Ligeras y Comercio (MILCO). En el mismo aparecen registradas 106 empresas con certificado de origen en la región zuliana, su ubicación geográfica y el sector económico en el cual opera su actividad exportadora. Donde un $35 \%$ corresponde a la industria tradicional, $34 \%$ a la industria intermedia, $21 \%$ a la industria mecánica y $10 \%$ a las actividades residuales.

En términos generales, se trata de unidades económicas cuya antigüedad promedio es menor de 20 años, gerenciadas por hombres, con buen nivel académico, con fuerte presencia de empresarios de origen extranjero y en etapa longeva, predomina la primera generación de propietarios, pues en la mayoría de los establecimientos no se ha producido el traspaso a la siguiente generación, no emplean reglas o principios que regulen el sistema familia-empresa. El $75 \%$ de estos negocios presentan falta de recursos financieros, tecnológicos, humanos y organizativos para acometer una expansión internacional. Manifiestan una elevada percepción del riesgo asociado al proceso de internacionalización, a pesar de ello rechazan la presencia de socios distintos a la familia empresaria, considerando la oportunidad de establecer alianzas estratégicas para incursionar en mercados internacionales.

Tomando en cuenta la realidad de las unidades económicas exportadoras del estado Zulia (occidente del país), se presenta un modelo prospectivo mediante el empleo de la metodología del análisis estructural. Este análisis es de gran importancia puesto que permite evaluar la situación de las empresas y delinear un 
direccionamiento a posibles escenarios favorables con el objetivo de generar ventajas competitivas en dicho sector, pues las empresas familiares son la columna vertebral de la economía, generan empleo e ingresos.

\section{METODOLOGÍA}

La construcción de un modelo teórico prospectivo obedece a la satisfacción de las necesidades de producción de conocimiento científico en un área determinada, es por ello, que según Martelo et al. (2016), así como Pukall y Calabrò (2014), este tiene la obligación de explicar un determinado universo de hechos y de predecir su comportamiento futuro, obtenido a través de procesos iterativos de reflexión y consulta que ayudan al refinamiento de visiones futuras.

En este sentido, la representación teórica de la realidad internacional de las empresas exportadoras familiares parte de la aplicación de la técnica prospectiva del análisis estructural, dicho instrumental permite pasar de la acción anticipada a la acción estratégica (Godet, 1999; Romero, 2009). Esta técnica consiste en analizar los fenómenos desde una perspectiva global, sistémica y dinámica, cuyo objeto es generar escenarios futuros, construidos no sólo con base a los datos del pasado sino teniendo en cuenta la evolución futura de las variables, así como el comportamiento de los actores implicados, de tal manera que se reduzca la incertidumbre y conduzca la acción presente hacia un futuro aceptable o deseado.

Se construyó y evaluó un modelo teórico de explicación de la dinámica de la internacionalización de la empresa familiar, mediante el empleo del análisis estructural. Para ello, fue necesaria la identificación de un conjunto inicial de variables que inciden en la situación problemática objeto de estudio. En este sentido, el procesamiento de la matriz de datos empíricos elaborada incluyó la eliminación de los valores constantes de las variables, el resultado fue sometido a un tratamiento de correlación bivariado, obteniéndose de esta forma la Ilamada Matriz de Coeficientes de Correlación Bivariada de Pearson. Estos valores se calculan considerando la siguiente regla: $0=0 ; 0.1$ a $0.350=1 ; 0.351$ a $0.60=2 ; 0.61$ a $1=3$. Luego esos coeficientes fueron recodificados atendiendo a un sistema de valores, para de esta manera hacer posible la introducción de los datos en la matriz de motricidad y dependencia del Programa MicMac. Esta matriz de correlaciones expresa el grado de asociación de las variables componentes del estudio entre sí misma y con las otras, permitiendo plasmar la realidad investigada como un sistema, cuyos elementos guardan relación de interdependencia (Tabla 1).

A través de la interrelación de ese conjunto de elementos se estableció la relación de motricidad y dependencia entre las variables, consiguiéndose el valor de cada una y la influencia que ejerce una sobre otras; esto fue posible mediante el Sistema de Matrices de Impactos Cruzados (SMIC). Una vez construida la matriz de motricidad y dependencia, se pudo conocer la fuerza explicativa de cada una de las fuentes de variabilidad a través de la asignación a cada uno de los elementos de un valor que oscila entre 0 y 3 . Se atribuyó valor cero (0) para la inexistencia de relación, uno (1) para aquella relación con bajo grado de motricidad y dependencia, dos (2) para un grado moderado y tres (3) para una intensidad alta. Estas asignaciones se llevaron a una tabla de doble entrada denominada Matriz de Impactos Cruzados, aplicada a una clasificación MICMAC. En las filas se indicaron las influencias directas de cada una de las variables sobre las otras, su total se denomina motricidad de la variable, mientras que en las columnas se representa el número de veces que la variable actúa sobre las otras, constituyéndose este valor en un indicador de dependencia de las mismas. Estas relaciones se expresan en el Tabla 2 que ilustra el nivel de motricidad y dependencia entre las variables del estudio.

La zonificación de las variables del modelo empírico se realizó considerando los valores porcentuales de motricidad y dependencia, calculados a partir de los resultados totales por filas y columnas, los cuales fueron ubicados en un gráfico que muestra cuatro zonas, a saber: Poder (variables con mayor motricidad y menor dependencia), este grupo es muy importante ya que influye sobre la mayoría y depende poco de ella, cualquier modificación de estas afecta a todo el sistema. Conflicto (variables con mayor motricidad y dependencia), son muy influyentes, pero también altamente vulnerables, ellas tienen un gran impacto sobre las demás, pero a su vez son muy afectadas por las otras. Salida (variables con alta dependencia y baja motricidad) y Problemas Autónomos (variables con baja dependencia y motricidad) es decir, que no son incidentes ni tampoco están influidas por las otras (Godet, 1999; Romero, 2009).

Para hallar los valores de las líneas vertical y horizontal que van a definir las cuatro zonas de relación gráfica de motricidad y dependencia en el plano cartesiano, se utiliza la fórmula de $x=100 / n$; siendo $n$ el número de variables consideradas en el estudio. Para efectos de la investigación realizada es: $100 / 50=2$. Tal y como se muestra en Figura 1, de un total de cincuenta (50) variables, el sistema permitió reducirlas, al tomar en cuenta sólo aquellas ubicadas en la zona de poder y de conflicto, dado que estas son las que presentan la más alta motricidad. 
Tabla 1: Matriz de análisis estructural del modelo empírico

\begin{tabular}{|c|c|c|c|c|c|c|c|c|c|c|c|c|c|c|c|c|c|c|c|c|c|c|c|c|c|}
\hline & $\begin{array}{l}\stackrel{7}{\pi} \\
\stackrel{0}{0} \\
\ddot{-}\end{array}$ & 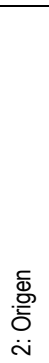 & 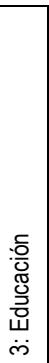 & 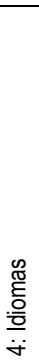 & 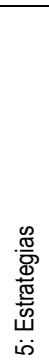 & $\begin{array}{l}\mathscr{0} \\
\stackrel{0}{0} \\
0 \\
00\end{array}$ & 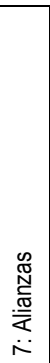 & $\begin{array}{c}\stackrel{8}{\mathscr{d}} \\
\stackrel{\mathscr{\omega}}{\alpha} \\
\dot{\infty}\end{array}$ & 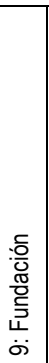 & 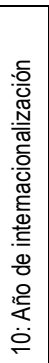 & 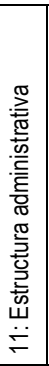 & 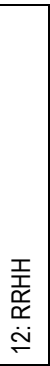 & 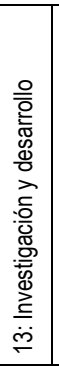 & 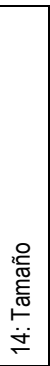 & 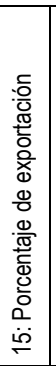 & 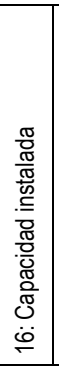 & 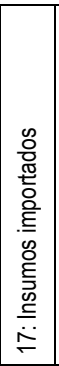 & 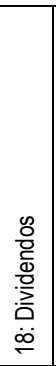 & 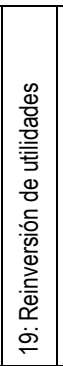 & 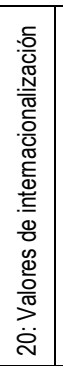 & 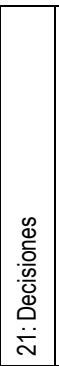 & 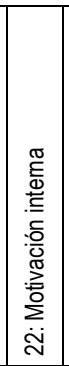 & $\begin{array}{c}0 \\
0 \\
0 \\
0 \\
.0 \\
\stackrel{0}{0} \\
\dot{0} \\
\tilde{N}\end{array}$ & 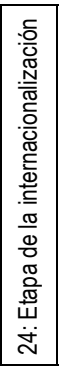 & 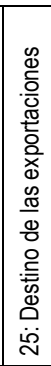 \\
\hline 1: Edad & 0 & 1 & 2 & 1 & 2 & 0 & 0 & 2 & 1 & 2 & 0 & 1 & 1 & 0 & 1 & 1 & 2 & 1 & 2 & 2 & 1 & 0 & 2 & 1 & 1 \\
\hline 2: Origen & 1 & 0 & 1 & 1 & 1 & 1 & 1 & 1 & 2 & 1 & 1 & 1 & 1 & 1 & 2 & 1 & 2 & 1 & 2 & 1 & 2 & 1 & 3 & 1 & 1 \\
\hline 3: Educación & 2 & 1 & 0 & 1 & 1 & 2 & 1 & 1 & 1 & 1 & 1 & 1 & 1 & 0 & 1 & 1 & 1 & 1 & 2 & 2 & 1 & 1 & 2 & 3 & 1 \\
\hline 4: Idiomas & 1 & 1 & 1 & 0 & 1 & 1 & 2 & 1 & 1 & 1 & 0 & 1 & 1 & 1 & 1 & 1 & 2 & 1 & 1 & 1 & 1 & 1 & 2 & 1 & 1 \\
\hline 5: Estrategias & 2 & 1 & 1 & 1 & 0 & 1 & 2 & 1 & 1 & 1 & 2 & 1 & 1 & 3 & 2 & 2 & 3 & 1 & 1 & 2 & 1 & 2 & 1 & 1 & 2 \\
\hline 6: Socios & 0 & 1 & 2 & 1 & 1 & 0 & 1 & 1 & 1 & 1 & 1 & 3 & 3 & 1 & 2 & 1 & 1 & 0 & 2 & 1 & 1 & 1 & 1 & 1 & 1 \\
\hline 7: Alianzas & 0 & 1 & 1 & 2 & 1 & 1 & 0 & 1 & 1 & 1 & 1 & 1 & 1 & 1 & 1 & 2 & 2 & 1 & 1 & 1 & 1 & 2 & 1 & 1 & 2 \\
\hline 8: Riesgo & 2 & 1 & 1 & 1 & 1 & 1 & 1 & 0 & 1 & 1 & 0 & 1 & 1 & 2 & 1 & 1 & 1 & 0 & 1 & 2 & 2 & 3 & 1 & 1 & 2 \\
\hline 9: Fundación & 1 & 2 & 1 & 2 & 1 & 1 & 1 & 1 & 0 & 1 & 3 & 2 & 2 & 1 & 2 & 1 & 1 & 1 & 1 & 2 & 2 & 1 & 1 & 1 & 2 \\
\hline $\begin{array}{l}\text { 10: Año de } \\
\text { internacionalización }\end{array}$ & 2 & 1 & 1 & 1 & 1 & 1 & 1 & 1 & 1 & 0 & 1 & 1 & 1 & 1 & 1 & 1 & 1 & 1 & 1 & 1 & 1 & 1 & 2 & 1 & 1 \\
\hline $\begin{array}{l}\text { 11: Estructura } \\
\text { administrativa }\end{array}$ & 0 & 1 & 1 & 1 & 2 & 1 & 1 & 0 & 3 & 1 & 0 & 2 & 2 & 2 & 3 & 1 & 1 & 1 & 1 & 2 & 1 & 1 & 1 & 0 & 1 \\
\hline 12: RRHH & 1 & 1 & 1 & 1 & 1 & 3 & 1 & 1 & 2 & 1 & 2 & 0 & 3 & 1 & 1 & 1 & 1 & 1 & 2 & 1 & 2 & 1 & 1 & 1 & 1 \\
\hline $\begin{array}{l}\text { 13: Investigació } \\
\text { desarrollo }\end{array}$ & 1 & 1 & 1 & 1 & 1 & 3 & 1 & 1 & 2 & 1 & 2 & 3 & 0 & 1 & 1 & 1 & 1 & 1 & 2 & 1 & 2 & 1 & 1 & 1 & 1 \\
\hline 14: Tamaño & 0 & 1 & 0 & 1 & 3 & 1 & 1 & 2 & 1 & 1 & 2 & 1 & 1 & 0 & 3 & 1 & 1 & 3 & 1 & 2 & 1 & 2 & 1 & 1 & 2 \\
\hline $\begin{array}{l}\text { 15: Porcentaje de } \\
\text { exportación }\end{array}$ & 1 & 2 & 1 & 1 & 2 & 2 & 1 & 1 & 2 & 1 & 3 & 1 & 1 & 3 & 0 & 1 & 0 & 2 & 1 & 2 & 2 & 1 & 1 & 1 & 1 \\
\hline $\begin{array}{l}\text { 16: Capacidad } \\
\text { instalada }\end{array}$ & 1 & 1 & 1 & 1 & 2 & 1 & 2 & 1 & 1 & 1 & 1 & 1 & 1 & 2 & 1 & 0 & 1 & 1 & 2 & 2 & 1 & 2 & 1 & 1 & 1 \\
\hline 17: Insumos imp & 2 & 2 & 1 & 2 & 3 & 1 & 2 & 1 & 1 & 1 & 1 & 1 & 1 & 1 & 0 & 1 & 0 & 1 & 3 & 1 & 2 & 1 & 1 & 1 & 1 \\
\hline 18: Dividendos & 1 & 1 & 1 & 1 & 1 & 0 & 1 & 0 & 1 & 1 & 1 & 1 & 1 & 3 & 2 & 1 & 1 & 0 & 1 & 0 & 1 & 1 & 1 & 1 & 1 \\
\hline $\begin{array}{l}\text { 19: Reinversión de } \\
\text { utilidades }\end{array}$ & 2 & 2 & 2 & 1 & 2 & 2 & 1 & 1 & 1 & 1 & 1 & 2 & 2 & 1 & 1 & 2 & 3 & 1 & 0 & 1 & 3 & 0 & 1 & 1 & 1 \\
\hline $\begin{array}{l}\text { 20: Valores de } \\
\text { internacionalización }\end{array}$ & 2 & 1 & 2 & 1 & 2 & 1 & 1 & 2 & 2 & 1 & 2 & 1 & 1 & 2 & 2 & 2 & 1 & 0 & 1 & 0 & 1 & 1 & 1 & 3 & 2 \\
\hline 21: Decisiones & 1 & 2 & 1 & 1 & 2 & 1 & 1 & 2 & 2 & 1 & 1 & 2 & 2 & 1 & 2 & 1 & 2 & 1 & 3 & 1 & 0 & 2 & 1 & 2 & 1 \\
\hline 22: Motivación interna & 0 & 1 & 1 & 1 & 2 & 1 & 2 & 3 & 1 & 1 & 1 & 1 & 1 & 2 & 1 & 2 & 1 & 1 & 0 & 1 & 2 & 0 & 1 & 1 & 3 \\
\hline lización & 2 & 3 & 2 & 2 & 1 & 1 & 1 & 1 & 1 & 2 & 1 & 1 & 1 & 1 & 1 & 1 & 1 & 1 & 1 & 1 & 1 & 1 & 0 & 2 & 1 \\
\hline $\begin{array}{l}\text { 24: Etapa de la } \\
\text { internacionalización }\end{array}$ & 1 & 1 & 3 & 1 & 1 & 1 & 1 & 1 & 1 & 1 & 0 & 1 & 1 & 1 & 1 & 1 & 1 & 2 & 1 & 3 & 2 & 1 & 2 & 0 & 2 \\
\hline $\begin{array}{l}\text { 25: Destino de las } \\
\text { exportaciones }\end{array}$ & 1 & 1 & 1 & 1 & 2 & 1 & 1 & 2 & 1 & 1 & 1 & 1 & 1 & 1 & 1 & 1 & 1 & 1 & 1 & 2 & 1 & 3 & 1 & 2 & 0 \\
\hline $\begin{array}{l}\text { 26: Actividad } \\
\text { económica }\end{array}$ & 2 & 2 & 1 & 2 & 1 & 1 & 1 & 1 & 2 & 2 & 3 & 1 & 1 & 2 & 2 & 1 & 1 & 2 & 1 & 1 & 2 & 1 & 1 & 1 & 1 \\
\hline 27: Cámaras & 2 & 1 & 1 & . & 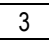 & 2 & 1 & 1 & 1 & 1 & 2 & 1 & 1 & 3 & 2 & 1 & 2 & 2 & 1 & 2 & 2 & 2 & 1 & 1 & 1 \\
\hline 28: Sector econó & 1 & 2 & 1 & 2 & 2 & 1 & 1 & 2 & 2 & 2 & 2 & 1 & 1 & 3 & 2 & 1 & 1 & 2 & 1 & 2 & 1 & 2 & 1 & 1 & 1 \\
\hline $\begin{array}{l}\text { 29: Medios de } \\
\text { información }\end{array}$ & 1 & 1 & 2 & 0 & 1 & 2 & 1 & 1 & 1 & 1 & 1 & 1 & 1 & 1 & 1 & 1 & 2 & 1 & 1 & 1 & 1 & 1 & 1 & 1 & 2 \\
\hline $\begin{array}{l}\text { 30: Fuentes de } \\
\text { financiamiento }\end{array}$ & 1 & 1 & 1 & 2 & 1 & 1 & 1 & 2 & 1 & 2 & 2 & 2 & 2 & 1 & 1 & 1 & 1 & 1 & 1 & 1 & 2 & 3 & 1 & 1 & 2 \\
\hline 31: Afiliación a cá & 2 & 1 & 1 & 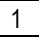 & 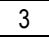 & 1 & 2 & 2 & 1 & 2 & 2 & 1 & 1 & 3 & 2 & 2 & 2 & 1 & 1 & 2 & 1 & 2 & 1 & 1 & 1 \\
\hline 32: Valores & 2 & 1 & 1 & 2 & 1 & 1 & 1 & 1 & 2 & 1 & 1 & 1 & 1 & 2 & 1 & 1 & 1 & 1 & 1 & 1 & 1 & 1 & 2 & 2 & 1 \\
\hline $\begin{array}{l}\text { 33: Recursos } \\
\text { necesarios }\end{array}$ & 1 & 1 & 1 & 1 & 2 & 1 & 1 & 1 & 1 & 1 & 1 & 1 & 1 & 2 & 1 & 2 & 3 & 2 & 3 & 1 & 3 & 1 & 1 & 1 & 1 \\
\hline $\begin{array}{l}\text { 34: Recursos } \\
\text { disponibles }\end{array}$ & 2 & 2 & 2 & 1 & 1 & 0 & 1 & 1 & 1 & 1 & 1 & 0 & 0 & 1 & 1 & 1 & 1 & 1 & 1 & 2 & 1 & 2 & 1 & 2 & 1 \\
\hline $\begin{array}{l}\text { 35: Incentivos } \\
\text { gubernamentales }\end{array}$ & 2 & 2 & 2 & 1 & 1 & 2 & 1 & 1 & 1 & 1 & 2 & 2 & 2 & 2 & 3 & 1 & 2 & 1 & 2 & 1 & 1 & 1 & 1 & 1 & 1 \\
\hline $\begin{array}{l}\text { 36: Vínculos con el } \\
\text { gobierno }\end{array}$ & 1 & 2 & 1 & 2 & 1 & 1 & 1 & 1 & 2 & 1 & 1 & 1 & 1 & 1 & 1 & 1 & 0 & 0 & 1 & 1 & 1 & 1 & 1 & 1 & 1 \\
\hline 37: Política cambiaria & 1 & 1 & 2 & 1 & 1 & 1 & 0 & 1 & 1 & 2 & 1 & 1 & 1 & 1 & 1 & 1 & 1 & $\angle$ & 1 & 2 & 1 & 1 & 1 & 1 & 0 \\
\hline $\begin{array}{l}\text { 38: Normativa } \\
\text { exportadora }\end{array}$ & 0 & 1 & 1 & 1 & 1 & 2 & 1 & 3 & 1 & 2 & 1 & 1 & 1 & 1 & 1 & 2 & 1 & 1 & 1 & 1 & 0 & 1 & 1 & 1 & 2 \\
\hline $\begin{array}{l}\text { 39: Condiciones de la } \\
\text { dinámica }\end{array}$ & 2 & 1 & 1 & 2 & 1 & 1 & 1 & 2 & 2 & 2 & 2 & 1 & 1 & 3 & 2 & 1 & 0 & 2 & 1 & 1 & 1 & 1 & 2 & 1 & 1 \\
\hline 40: Infraestructura & 0 & 1 & 1 & 1 & 1 & 2 & 1 & 1 & 1 & 2 & 1 & 1 & 1 & 2 & 1 & 1 & 1 & 1 & 3 & 1 & 2 & 1 & 1 & 1 & 1 \\
\hline $\begin{array}{l}\text { 41: Acuerdos } \\
\text { económicos }\end{array}$ & 1 & 2 & 1 & 1 & 1 & 1 & 1 & 2 & 2 & 1 & 1 & 1 & 1 & 1 & 1 & 1 & 1 & 1 & 1 & 1 & 1 & 1 & 1 & 1 & 1 \\
\hline $\begin{array}{l}\text { 42: Medidas del } \\
\text { gobierno }\end{array}$ & 3 & 1 & 1 & 1 & 2 & 1 & 1 & 1 & 1 & 1 & 2 & 1 & 1 & 1 & 1 & 1 & 1 & 2 & 1 & 2 & 1 & 1 & 2 & 1 & 1 \\
\hline
\end{tabular}


Tabla 1: continuación

\begin{tabular}{|c|c|c|c|c|c|c|c|c|c|c|c|c|c|c|c|c|c|c|c|c|c|c|c|c|c|}
\hline & 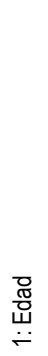 & $\begin{array}{l}\bar{D} \\
\text { D্ } \\
\text { Dे } \\
\text { ì }\end{array}$ & 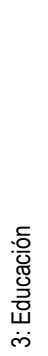 & 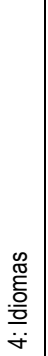 & 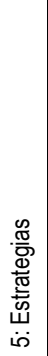 & $\begin{array}{l}\mathscr{0} \\
\ddot{0} \\
0 \\
\ddot{0}\end{array}$ & 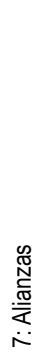 & 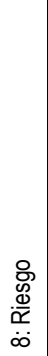 & 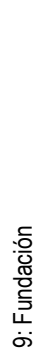 & 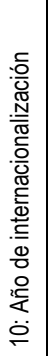 & 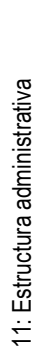 & $\begin{array}{l}\text { 폼 } \\
\text { 똠 } \\
\stackrel{ }{ }\end{array}$ & 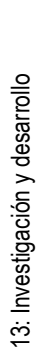 & 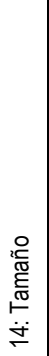 & 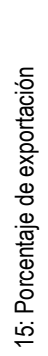 & 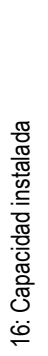 & 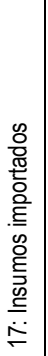 & 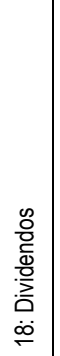 & 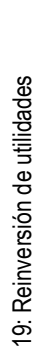 & 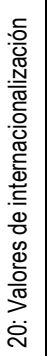 & 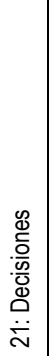 & 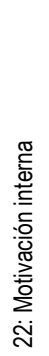 & 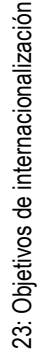 & 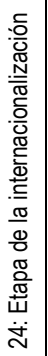 & 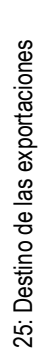 \\
\hline $\begin{array}{l}\text { 43: Preferencias de } \\
\text { mercado }\end{array}$ & 1 & 1 & 2 & 1 & 1 & 1 & 1 & 2 & 2 & 1 & 1 & 1 & 1 & 1 & 1 & 1 & 1 & 1 & 1 & 3 & 1 & 2 & 1 & 3 & 2 \\
\hline $\begin{array}{l}\text { 44: Obstáculos d la } \\
\text { dinámica país }\end{array}$ & 0 & 1 & 1 & 1 & 2 & 1 & 1 & 2 & 1 & 1 & 3 & 1 & 1 & 1 & 2 & 1 & 2 & 1 & 0 & 1 & 1 & 1 & 1 & 1 & 1 \\
\hline $\begin{array}{l}\text { 45: Variables } \\
\text { económicas }\end{array}$ & 1 & 1 & 1 & 1 & 1 & 1 & 1 & 1 & 1 & 1 & 1 & 1 & 1 & 1 & 1 & 3 & 1 & 1 & 1 & 2 & 1 & 1 & 2 & 1 & 1 \\
\hline $\begin{array}{l}\text { 46: Generación que } \\
\text { dirige }\end{array}$ & 2 & 2 & 1 & 2 & 1 & 2 & 1 & 1 & 3 & 1 & 1 & 1 & 1 & 1 & 1 & 1 & 1 & 1 & 1 & 1 & 2 & 1 & 1 & 1 & 1 \\
\hline 47: Protocolo & 2 & 2 & 1 & 2 & 1 & 2 & 1 & 1 & 3 & 1 & 1 & 1 & 1 & 1 & 1 & 1 & 1 & 1 & 1 & 1 & 2 & 1 & 1 & 1 & 1 \\
\hline $\begin{array}{l}\text { 48: Trabajadores } \\
\text { familiares }\end{array}$ & 1 & 1 & 1 & 1 & 1 & 3 & 1 & 1 & 1 & 2 & 1 & 2 & 2 & 1 & 1 & 1 & 1 & 1 & 2 & 1 & 2 & 1 & 2 & 1 & 0 \\
\hline 49: Propiedad familiar & 1 & 2 & 2 & 1 & 1 & 1 & 1 & 2 & 1 & 1 & 1 & 1 & 1 & 1 & 1 & 2 & 1 & 1 & 1 & 2 & 2 & 2 & 1 & 2 & 1 \\
\hline 50: Sucesión & 2 & 1 & 1 & 1 & 2 & 1 & 1 & 1 & 1 & 1 & 1 & 1 & 1 & 3 & 2 & 1 & 0 & 2 & 1 & 1 & 1 & 2 & 3 & 1 & 2 \\
\hline
\end{tabular}

Tabla 2: Relación de motricidad y dependencia del modelo empírico

\begin{tabular}{|c|c|c|c|c|c|}
\hline$N^{\circ}$ & Variable & $\begin{array}{l}\text { Número total de } \\
\text { filas }\end{array}$ & $\begin{array}{c}\text { Porcentaje de } \\
\text { motrocidad }\end{array}$ & $\begin{array}{l}\text { Número total de } \\
\text { columnas }\end{array}$ & $\begin{array}{l}\text { Porcentaje de } \\
\text { dependencia }\end{array}$ \\
\hline 1 & Edad del empresario familiar & 60 & $1,89 \%$ & 61 & $1,92 \%$ \\
\hline 2 & Origen geográfico & 65 & $2,05 \%$ & 65 & $2,04 \%$ \\
\hline 3 & Nivel educativo & 61 & $1,92 \%$ & 61 & $1,92 \%$ \\
\hline 4 & Idiomas que habla el empresario & 59 & $1,86 \%$ & 60 & $1,88 \%$ \\
\hline 5 & Estrategia de internacionalización & 69 & $2,17 \%$ & 71 & $2,23 \%$ \\
\hline 6 & Nuevos socios & 63 & $1,98 \%$ & 62 & $1,95 \%$ \\
\hline 7 & Alianzas de la empresa familiar & 52 & $1,64 \%$ & 53 & $1,66 \%$ \\
\hline 8 & Riesgo de la internacionalización & 63 & $1,98 \%$ & 63 & $1,98 \%$ \\
\hline 9 & Fundación de la empresa & 71 & $2,23 \%$ & 68 & $2,14 \%$ \\
\hline 10 & Año de internacionalización & 60 & $1,89 \%$ & 60 & $1,88 \%$ \\
\hline 11 & Estructura administrativa & 66 & $2,08 \%$ & 65 & $2,04 \%$ \\
\hline 12 & Recursos humanos calificados & 59 & $1,86 \%$ & 59 & $1,85 \%$ \\
\hline 13 & Investigación y desarrollo & 59 & $1,86 \%$ & 59 & $1,85 \%$ \\
\hline 14 & Tamaño de la empresa & 73 & $2,30 \%$ & 73 & $2,29 \%$ \\
\hline 15 & Porcentaje de producto exportado & 68 & $2,14 \%$ & 68 & $2,14 \%$ \\
\hline 16 & Porcentaje de uso de la capacidad instalada & 61 & $1,92 \%$ & 60 & $1,88 \%$ \\
\hline 17 & Porcentaje de uso de insumos importados & 60 & $1,89 \%$ & 61 & $1,92 \%$ \\
\hline 18 & Reparto de dividendos & 55 & $1,73 \%$ & 57 & $1,79 \%$ \\
\hline 19 & Porcentaje de reinversión de utilidades & 65 & $2,05 \%$ & 64 & $2,01 \%$ \\
\hline 20 & Valores de la internacionalización & 71 & $2,23 \%$ & 70 & $2,20 \%$ \\
\hline 21 & Toma de decisiones en la empresa & 70 & $2,20 \%$ & 69 & $2,17 \%$ \\
\hline 22 & Motivación interna & 65 & $2,05 \%$ & 65 & $2,04 \%$ \\
\hline 23 & Objetivos de la internacionalización & 62 & $1,95 \%$ & 63 & $1,98 \%$ \\
\hline 24 & Etapa de la internacionalización & 60 & $1,89 \%$ & 60 & $1,88 \%$ \\
\hline 25 & Destinos de las exportaciones & 60 & $1,89 \%$ & 61 & $1,92 \%$ \\
\hline 26 & Sector de actividad económica & 71 & $2,23 \%$ & 72 & $2,26 \%$ \\
\hline 27 & Cámaras a las cuales está afiliada & 71 & $2,23 \%$ & 72 & $2,26 \%$ \\
\hline 28 & Sector económico & 76 & $2,39 \%$ & 77 & $2,42 \%$ \\
\hline 29 & Medios de información para internacionalización & 57 & $1,79 \%$ & 56 & $1,76 \%$ \\
\hline 30 & Fuentes de financiamiento & 64 & $2,01 \%$ & 64 & $2,01 \%$ \\
\hline 31 & Afiliación a cámaras & 69 & $2,17 \%$ & 68 & $2,14 \%$ \\
\hline 32 & Valores de la empresa & 65 & $2,05 \%$ & 62 & $1,95 \%$ \\
\hline
\end{tabular}


Tabla 2: continuación

\begin{tabular}{|l|l|c|c|c|c|}
\hline 33 & Recursos necesarios & 67 & $2,11 \%$ & 67 & $2,10 \%$ \\
\hline 34 & Recursos disponibles & 58 & $1,83 \%$ & 58 & $1,82 \%$ \\
\hline 35 & Uso de incentivos gubernamentales & 71 & $2,23 \%$ & 72 & $2,26 \%$ \\
\hline 36 & Vínculos con el gobierno & 61 & $1,92 \%$ & 63 & $1,98 \%$ \\
\hline 37 & Efecto de la política cambiaria & 57 & $1,79 \%$ & 53 & $1,66 \%$ \\
\hline 38 & Normativa exportadora & 58 & $1,83 \%$ & 59 & $1,85 \%$ \\
\hline 39 & Condiciones de la dinámica & 71 & $2,23 \%$ & 71 & $2,23 \%$ \\
\hline 40 & Estado de la infraestructura & 61 & $1,92 \%$ & 59 & $1,85 \%$ \\
\hline 41 & Empleo de acuerdos económicos & 59 & $1,86 \%$ & 60 & $1,88 \%$ \\
\hline 42 & Medidas del gobierno & 61 & $1,92 \%$ & 62 & $1,95 \%$ \\
\hline 43 & Preferencias de mercado & 66 & $2,08 \%$ & 67 & $2,10 \%$ \\
\hline 44 & Obstáculos de la dinámica del país & 56 & $1,76 \%$ & 56 & $1,76 \%$ \\
\hline 45 & Impacto de las variables económicas & 56 & $1,76 \%$ & 56 & $1,76 \%$ \\
\hline 46 & Generación que dirige la empresa & 66 & $2,08 \%$ & 66 & $2,07 \%$ \\
\hline 47 & Existencia de protocolo en la empresa & 67 & $2,11 \%$ & 68 & $2,14 \%$ \\
\hline 48 & Área de la empresa donde trabajan los familiares & 65 & $2,05 \%$ & 62 & $1,95 \%$ \\
\hline 49 & Porcentaje de propiedad familiar en la empresa & 64 & $2,01 \%$ & 65 & $2,04 \%$ \\
\hline 50 & Planificación de la sucesión & 63 & $1,98 \%$ & 64 & $2,01 \%$ \\
\hline & Totales & 3177 & $100 \%$ & 3177 & $100 \%$ \\
\hline
\end{tabular}

\section{Direct influence/dependence map}

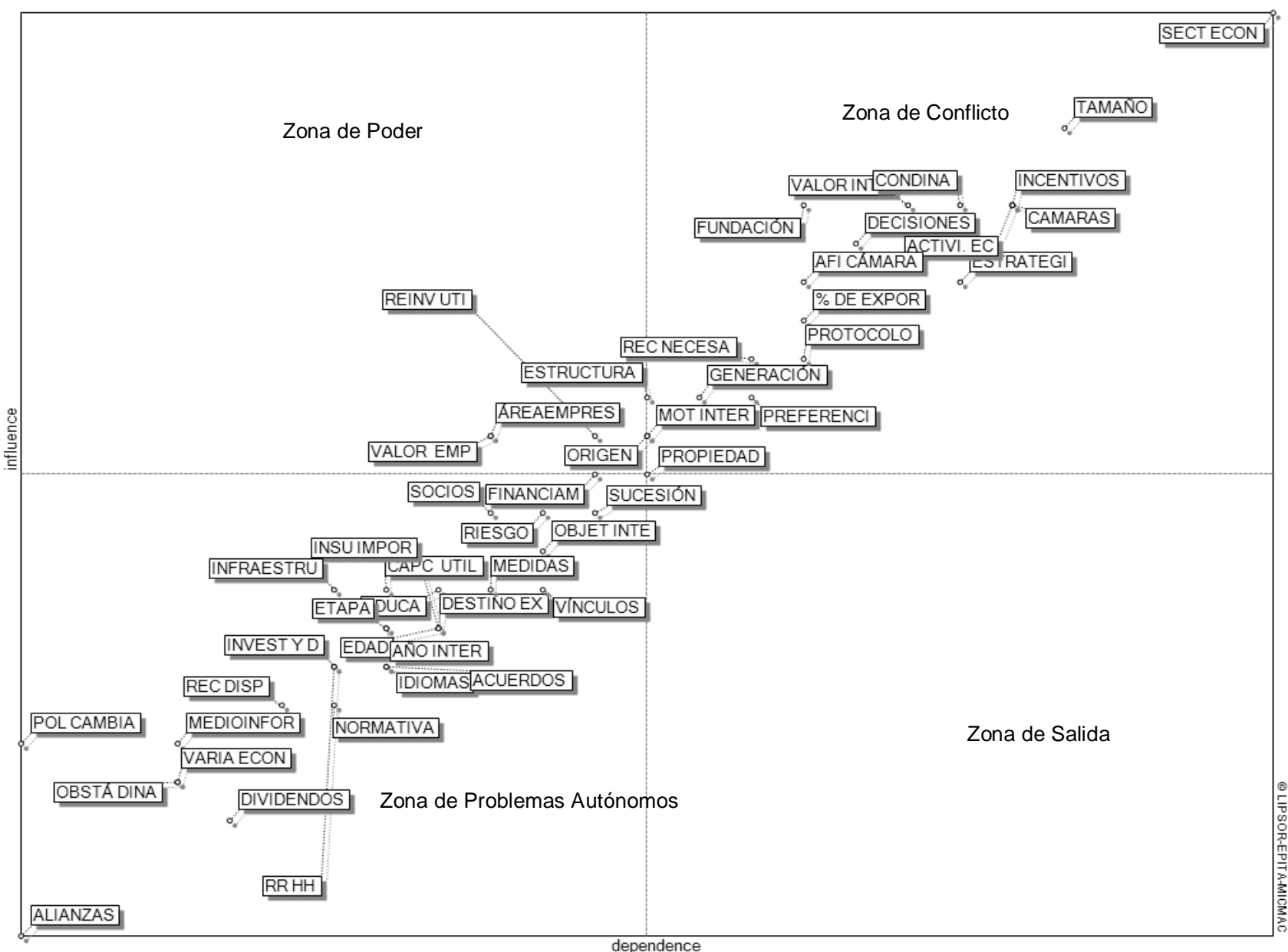

Fig 1: Zonificación de las variables 


\section{RESULTADOS Y DISCUSIÓN}

Como resultado de la aplicación de la técnica prospectiva del análisis estructural, se obtuvo un modelo representativo de las empresas familiares exportadoras de la región zuliana - Venezuela, modelo que corresponde a uno de naturaleza empírica, en el cual los datos que alimentaron su base fueron producto de los resultados obtenidos en el estudio de campo. A partir de allí, se conformó un sistema contentivo de cincuenta variables, discriminadas en internas y externas, las primeras, están referidas a todos aquellos elementos de las empresas que determinan sus ventajas competitivas, expresadas en recursos y capacidades que están bajo control empresarial. Las segundas, consideran los elementos del entorno de la empresa no controlables por ésta, que afectan desde afuera el comportamiento actual y futuro de los negocios familiares. Posteriormente, para determinar cuáles de las variables son críticas para el comportamiento del sistema, se derivó la Matriz de Impacto Cruzado que permitió ilustrar el nivel de motricidad y dependencia de las variables implícitas en el estudio; ubicándolas en un plano cartesiano que define cuatro zonas de relación (poder, conflicto, problemas autónomos y salida). Dada la gran cantidad de variables presentes en el modelo, el análisis se centró en las situadas en la zona de poder, ya que son las que más afectan el sistema. Al respecto, es necesario acotar que el resultado de éstas se corresponde más con la categoría de análisis denominada variables internas, como: Estructura administrativa, propiedad en manos de la familia, área en la que trabajan los familiares en la empresa, valores familiares en la empresa, origen del empresario, motivación interna para exportar, fuentes de financiamiento y reinversión de utilidades. En la Figura 2 se aprecia de manera gráfica esta perspectiva.

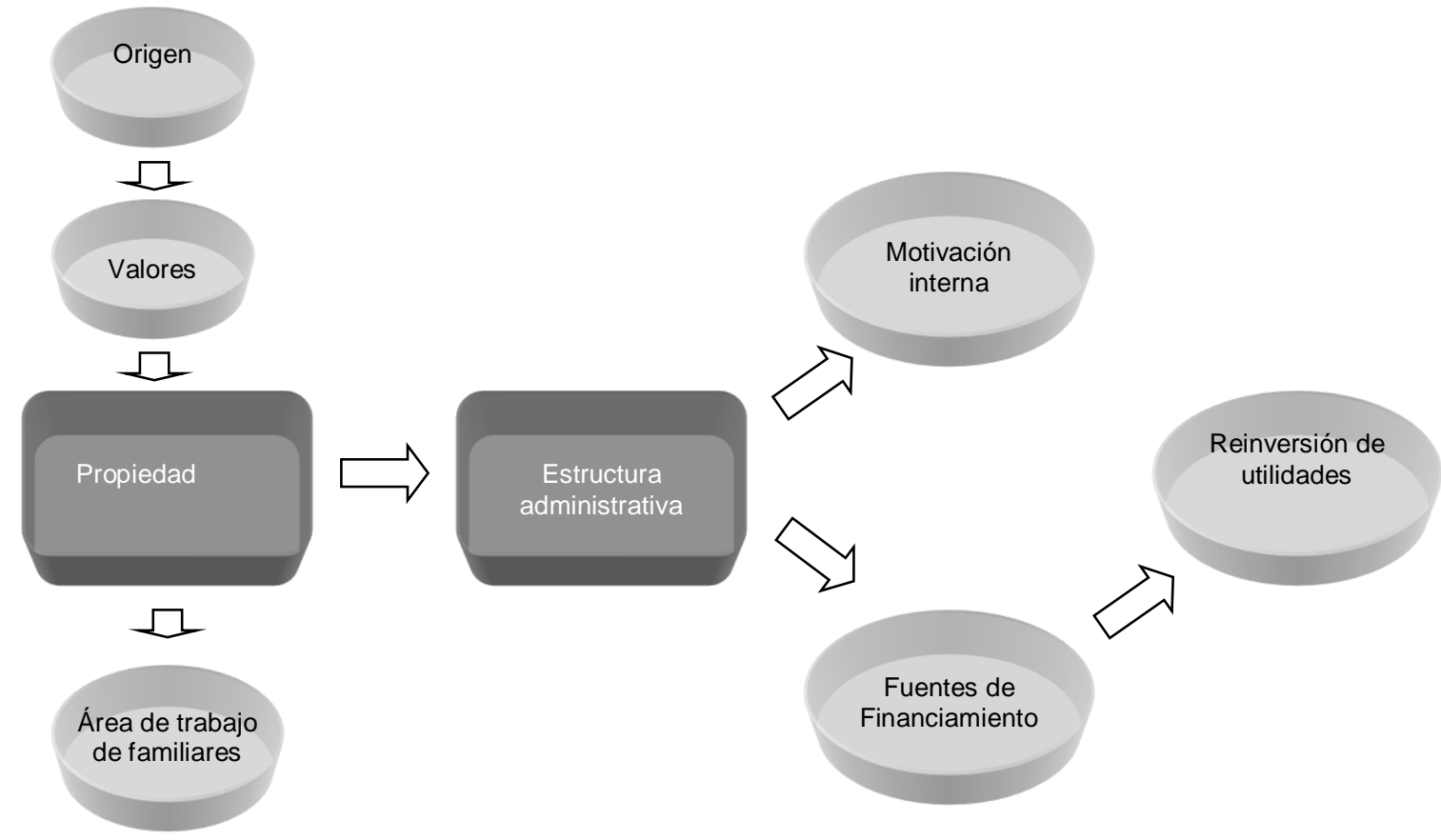

Fig 2: Modelo prospectivo de la dinámica exportadora de la empresa familiar

Es menester subrayar, que la manera como están organizadas las variables en la gráfica fue producto de la aplicación del método estadístico del Análisis Factorial, que llevó a determinar el examen de sus componentes principales y el análisis de las correlaciones existente entre ellas. En tal sentido, el lugar que ocupa cada una de estas obedeció al resultado de su fuerza explicativa. En términos generales, el hecho de que todas las variables pertenezcan a los llamados variables internas permitió inferir que la visión que los empresarios tienen acerca de la dinámica futura de sus exportaciones descansa más en aspectos sobre los cuales pueden ejercer el poder de acción y control. Básicamente, prefieren confiar en la eficacia de su gestión, en su capacidad productiva y en los recursos disponibles. En efecto, se puede visualizar que existen dos variables sobre la cual gira esta visión: La propiedad y la existencia de una estructura administrativa para el desarrollo de la internacionalización, aspectos que fueron determinados de acuerdo con el análisis estadístico de sus componentes principales.

\section{Propiedad}

La propiedad de la familia en la empresa resultó ser una de las variables de poder más influyente en este enfoque. Este aspecto se puede interpretar como el deseo que tienen estos empresarios de que sus organizaciones sigan siendo de naturaleza familiar, donde el poder accionario y la toma de decisiones 
descansen en el grupo familiar que la dirige. De acuerdo con esta óptica, demarcada por una cultura conservadora y poco arriesgada, es necesario mantener el control de la empresa, pues no se debe olvidar que la familia satisface sus necesidades materiales a través de ésta, al mismo tiempo que constituye el patrimonio de la próxima generación de propietarios. Esta variable está referida a la estructura accionaria de la empresa familiar, es decir, la manera como están distribuidas las acciones entre los socios. En el ámbito de los negocios exportadores zulianos esta disposición se concentra en una sola familia, específicamente es el fundador quien controla el cien por ciento de ésta, observándose además una alta participación del grupo familiar en la dirección de la empresa, ocupando puestos estratégicos como señala Alayo et al. (2019).

Cabe destacar, que se percibe una clara tendencia a seguir detentando la familia el poder del capital, ello explicado por la negativa puesta en evidencia de admitir socios distintos al grupo parentelar a pesar de las carencias de recursos, inferido en el estudio empírico. La razón esgrimida está relacionada con la naturaleza de estas organizaciones de constituirse en un legado para las próximas generaciones (Hernández et al., 2015), así como también en el temor de que decisiones de miembros ajenos a la familia vulneren la estabilidad material de esta última. Sin embargo, en la medida en que la empresa va consolidándose en el tiempo, y se produce el cambio generacional se complejiza su estructura accionaria al multiplicarse el número de socios y cambiar su relación de parentesco. Tal situación refleja la incorporación de una diversidad de intereses que obviamente compromete la estabilidad de la empresa y exige tomar acciones que permitan una transición exitosa hacia la segunda generación de propietarios, tal y lo como plantea Acosta et al. (2019); escenario factible en un mediano a corto plazo en el contexto estudiado, cuidando el liderazgo, la cultura familiar empresaria y la relación familia - empresa.

Por otra parte, hay que considerar que estos empresarios revelaron que se sienten sometidos a ciertas presiones del ámbito externo en el cual se desenvuelve la empresa, concretamente la esfera política, jurídica y económica, los llena de incertidumbre según De la Hoz-Dominguez et al. (2019), y los obliga a resguardar la propiedad en el seno familiar. La amenaza de expropiaciones, confiscaciones y debilitamiento de la propiedad privada es un claro ejemplo de tales presiones y explican, en gran parte, el por qué esta variable resultó ser percibida de manera estratégica por los dueños de negocios familiares encuestados.

Al colocar el acento en el área de la propiedad, se exaltan los valores familiares sobre los cuales se sustenta la empresa. En este punto los empresarios reflexionan que para fortalecer el negocio de la familia es necesaria la unión entre sus miembros para hacer frente a las circunstancias del mercado y del entorno en general en que se desenvuelve la actividad exportadora. Los valores de la empresa familiar constituyen un aspecto fundamental en este modelo empresarial en la opinión de Garza et al. (2011), ya que la identificación y la socialización de los principios presentes en los fundadores a los miembros de la siguiente generación de propietarios garantizan la supervivencia de la misma en el futuro. Este elemento se torna un tanto estratégico, sobre todo si se considera que en la mayor parte de las empresas estudiadas no se ha producido el traspaso de la batuta a la siguiente generación.

Lo esbozado con anterioridad, se inscribe dentro del planteamiento formulado por Cóndor et al. (2018), así como por Liang et al. (2014), quienes señalan que es el líder fundador de la empresa el elemento clave, pues su sistema de valores, en primer lugar, influencia la creación de la empresa y luego, condiciona su evolución y futuro a través de la transmisión de valores al ámbito empresarial y familiar. Es así, como el poder de la familia en la empresa se manifesta con lazos más fuerte que los intereses económicos. Esa fuerza se expresa a través de valores que guían la conducta de sus miembros. Cabe destacar, que en el caso las empresas objeto de análisis, los valores predominantes que arrojó el estudio de campo fueron: la responsabilidad, la confianza y el compromiso. Este punto se considera de suma importancia ya que la identificación de estos es la primera tarea a tener en cuenta.

Cuando los valores no son identificados no pueden ser claramente transmitidos, y en consecuencia, la cultura empresarial se debilita acarreando con el tiempo un posible cierre del negocio. Vale la pena resaltar lo acotado por los profesores Tapies y Ward (2008) al señalar la importancia del sistema de valores en la continuidad de este tipo de empresas. Al respecto, mencionan que este sistema es propio de cada familia, por lo que no pueden existir dos empresas con la misma estructura, aunque podrían ser muy parecidos debido a que tanto la familia como la empresa están influidas por los valores presentes de la comunidad o localidad donde se encuentran insertas. En el sistema de valores subyace una serie de principios que motivan y regulan la acción empresarial, estas pautas permanecen casi inalterables en el tiempo, sólo se adaptan debido a situaciones del entorno y se transfieren a los descendientes o herederos. En la medida en que valores familiares se fortalecen, la propiedad familiar también se robustece; sin embargo, uno de los errores que comúnmente cometen estas organizaciones es el de descuidar la transmisión de los principios que dieron el impulso al espíritu emprendedor y el no desarrollar órganos de gobierno indispensables cuando la familia y la empresa crecen y se hace necesaria la transición a la nueva generación de propietarios. 
Los órganos de gobierno constituyen herramientas que permiten atenuar los efectos del sistema familiar en la empresa, el Protocolo Familiar y el Consejo de Familia promueven la unidad y el compromiso entre los miembros, al mismo tiempo que limitan la influencia de los familiares cuando estos ponen en peligro los intereses económicos del negocio, tal como lo enuncian Hernández et al. (2015). La necesidad de implementar estos mecanismos se hace más pronunciada cuando las empresas entran en una fase de desarrollo internacional, que exige que estas respondan de manera más profesional, es decir, que se ajusten a los requerimientos de un entorno global.

En las relaciones puestas de manifiesto en la Figura 1, se evidencia que la dinámica exportadora de la empresa familiar zuliana está condicionada a la actitud que el empresario tiene frente al proceso de internacionalización, y donde la cultura bajo la cual se ha formado es determinante, un rasgo que sobresale en la formación de dicha identidad está relacionado con su origen nacional o extranjero. En tal sentido, se podría derivar que el hecho de ser descendiente o nacido en el extranjero hace que desde el punto de vista psicológico y cultural esté más predispuesto a asumir el riesgo que implica la actividad internacional según indica Clark et al. (2018), pues la condición de extranjero hace al empresario menos temeroso y/o más arriesgado, porque el aporte de otras culturas le brinda mayor seguridad y conocimiento en el emprendimiento de negocios foráneos.

Por otra parte, la elevada presencia de empresarios de origen extranjero devela que existen valores en ellos que los impulsa a desarrollar actividades productivas, así como abordar el comercio internacional, y que son propios de culturas que fomentan la competitividad entre sus miembros. Sobre este aspecto, es necesario indagar en futuras investigaciones, si en efecto, los valores del venezolano están orientados a promover el espíritu de libre empresa, o si, por el contrario, los principios se identifican más con aquellos que sólo exaltan el trabajo asalariado como valor para la sociedad.

Las características que definen el tipo de familia que gestiona las empresas exportadoras de la región zuliana corresponde más a la llamada familia nuclear, compuesta por padres e hijos, aunque también participan miembros de la familia extendida. Lo expuesto se deriva del hecho que estas empresas se encuentran transitando la primera generación de propietarios, y en un corto plazo se traspasará la batuta del fundador a sus hijos, ampliándose la propiedad accionaria, así como los miembros de la familia que participan en el negocio. Esta participación debe hacerse en las mejores condiciones para que redunde en una buena gestión de la empresa, como expresa Hurmerinta et al. (2016), de manera que el desempeño de la familia debe evaluarse con los mismos criterios utilizados para los empleados no familiares, y no sólo considerar el rango familiar, o en el peor de los casos incurrir en la falta de supervisión de sus actividades, tal y como lo mencionan en su trabajo Hernández y Portillo (2016). La inserción de los miembros del grupo familiar a la empresa debe estar orientada a desarrollar las competencias suficientes para ejercer los cargos, sobre todo los de tipo directivo que exigen una sólida formación profesional y experiencia para ejecutar sus funciones como lo hace notar Hernández y Portillo (2016). Obviamente, será necesario establecer normas que regulen la entrada a la organización y el desempeño de los mismos, como, por ejemplo: el establecimiento de políticas salariales en función de las responsabilidades del puesto, el diseño de planes de carrera ajustado a las necesidades del negocio, entre otras.

Lo apuntado anteriormente se reflejó en el modelo empírico derivado del análisis estructural, que resaltó el papel preponderante que tienen los miembros del grupo familiar que trabajan en la empresa como factor para potenciar la propiedad en manos de la familia, con este fin se subrayó su participación en las distintas áreas estratégicas de la organización. Siendo uno de estos espacios la actividad exportadora, la cual deberá ser manejada por estos, pero que necesitará de un mayor nivel de formalidad en cuanto a la estructura administrativa y un elevado nivel de conocimiento del personal en lo concerniente a trámites, requisitos, incentivos, mercados, entre otros aspectos. En consecuencia, para hacer sostenible la organización en los mercados internacionales se deberá dedicar esfuerzos a la formación progresiva y continua del recurso humano, sea este de tipo familiar y no familiar. A criterio de Marín et al., (2018), todo proceso formativo mejora las competencias en una materia específica y amplía la visión integral de conocimientos, impactando positivamente en destrezas, habilidades, valores y actitudes de desempeño.

\section{Estructura administrativa para las operaciones de exportación}

La actividad exportadora constituye una fuente de crecimiento y consolidación de muchas empresas, ella exige una adecuada planeación de recursos para garantizar su éxito en el mercado internacional (Buccieria et al., 2020; Metsola et al., 2020). En tal sentido, la presencia de un departamento o unidad administrativa encargada de coordinar todas las acciones necesarias para las ventas al exterior es un paso importante para su fortalecimiento y desarrollo ulterior. Cabe destacar, que esta estructura no existe en las empresas estudiadas, dado que la misma funciona como un complemento de otros departamentos operativos como finanzas y administración, que han centralizado los procedimientos relativos a las exportaciones producto del bajo volumen de ventas registradas en el mercado internacional. 
Asimismo, algunas empresas familiares han dejado en manos de intermediarios sus operaciones internacionales a través de la contratación de comercializadoras o agentes de exportación; en consecuencia, se han conformado con cuotas de participación de mercado muy bajas a pesar de considerar que el producto vendido es de excelente calidad y de que el mismo goza de una demanda creciente en el escenario mundial. El panorama anterior requiere que en el seno de estas empresas se produzcan cambios en el futuro que conduzcan al fortalecimiento de la actividad exportadora mediante la creación de una sección o departamento encargado para este fin. Con ello se persigue dar una mayor formalidad y organización a las operaciones de comercio exterior, lo cual demandará la modernización tecnológica y la capacitación de recursos humanos en el área internacional.

Todo empresario que se decida enfrentar el proyecto exportador con posibilidades de éxito deberá asignar los recursos necesarios. Bien es sabido que las transacciones con el exterior presentan cierto grado de complejidad y riesgos que necesitan de la dedicación y atención de profesionales expertos. Aunque la literatura consultada acerca del tema indica que en el caso de las empresas familiares la gestión de comercio exterior recae en el dueño de la empresa (Kampouri et al., 2017; Niittymies y Pajunen, 2020), es conveniente que dado la complejidad señalada en las funciones, se requiera de un gerente o encargado que pertenezca preferiblemente al ámbito familiar; de esta manera se preserva la toma de decisiones en áreas estratégicas del negocio, por lo que, se torna pertinente la formación de un profesional en el plano de legislación aduanera, trámites y acuerdos internacionales, entre otros aspectos.

Entre las numerosas funciones que ha de cumplir esta unidad de negocios internacionales están: Estudiar la demanda potencial del producto en el mercado internacional; seleccionar y contratar agentes o distribuidores; acceder a programas de financiamiento para el proceso exportador y a programas de incentivos para la promoción de exportaciones; conocer y cumplir con todas las reglamentaciones tanto de origen como de destino; coordinar las operaciones bancarias e impositivas relacionadas con la exportación y dar seguimiento al cobro de lo exportado.

Concretamente en Venezuela, la tramitación de las exportaciones requiere de una cantidad considerable de requisitos al igual que en la mayoría de los países, lo cual va a depender del tipo de mercancía a exportar. A continuación, se presentan algunos de los permisos, registros y certificados previos a la exportación. Entre ellos destaca: Registro del exportador, certificado de origen, certificado sanitario, registro de la empresa ante el Ministerio de Energía y Minas (MEM), la clasificación arancelaria del producto de acuerdo con el Sistema Armonizado (SA). Adicionalmente, se determina si las mercancías deben pagar algún tipo de impuesto y si satisfacen los múltiples requisitos que en su conjunto suelen llamarse barreras no arancelarias.

Como se observa son muchos los aspectos que deben manejar los encargados de esta unidad administrativa, por lo que se requiere de una sólida formación profesional de este personal, aunque por lo general, la empresa familiar no dispone de suficientes familiares calificados en comercio internacional, significando con ello una gran debilidad a la hora de abordar este mercado; por esta razón, desde el punto de vista estratégico, la formación de algún miembro del grupo familiar es vital para hacer frente a tal problemática. La consolidación de una estructura administrativa que conduzca la actividad exportadora en las empresas familiares zulianas requerirá como se mencionó anteriormente, de la afluencia de una serie de recursos de tipo material, tecnológico y humano; para ello la organización proveerá los fondos para su financiamiento. Este aspecto se considera un punto álgido en el caso de los negocios de familia, pues como comentan Romero et al. (2015), existe un rechazo por el empleo de fuentes de financiamiento externas, que las inclina sólo a manejarse con capital propio, esta razón impone grandes límites al desarrollo de la actividad internacional.

Su dificultad para alcanzar una mayor participación en el comercio internacional se focaliza en el hecho de ejecutar políticas financieras que se caracterizan por el uso de prácticas conservadoras de endeudamiento. Aznarez y Vilaseca (2004) explican que las razones por las cuales se da tal conducta obedecen a los siguientes aspectos: 1) Falta de diversificación del patrimonio familiar. El capital se origina de la concentración de la riqueza familiar, por tanto, tenderán a no comprometerlo. 2) Relación entre capital y rentas laborales. Por lo general, en este tipo de empresas los miembros de la familia mantienen no sólo una participación accionaria, sino que también son trabajadores o directivos. En tal sentido, los costos de la bancarrota producto de un mayor nivel de endeudamiento tienen un impacto en la situación de la familia tanto como accionista como trabajador. 3) Pérdida efectiva del control de la empresa por el componente familiar a favor de la institución o accionista que provee el financiamiento. 4) Situación fiscal, la desviación de gastos de la empresa con destino al sector familiar puede hacer ver una disminución en el beneficio operativo del negocio y de su capacidad para hacer frente a nuevas cargas financieras, así como también de sus posibilidades de endeudamiento.

Este comportamiento explica por qué la empresa familiar opta por centrar su actividad en sectores exportadores relacionados con la industria tradicional, pues implica un menor grado de desarrollo tecnológico, 
ya que incorpora menos cantidad de factor capital dentro de su estructura productiva, lo que la obliga a trabajar en función de la explotación de las ventajas comparativas de la región, exportando productos con bajo valor agregado, con procesos que implican el empleo de recursos humanos de escasa calificación y de algunas materias primas con exigua transformación.

\section{DISCUSIÓN FINAL}

La premisa fundamental del modelo es la de garantizar el control de la familia sobre la empresa, así, el crecimiento de la actividad internacional se afianzará sobre todo en los fondos financieros propios, concretamente, la reinversión de utilidades constituye la vía fundamental para sufragar los gastos derivados de la expansión internacional. Por lo que se asume la conducción del proceso de manera más lenta que en el caso de las empresas no familiares, ya que los fondos provenientes de terceras fuentes son usados con cautela. No obstante, ante la falta de recursos financieros es conveniente explorar otras fuentes que involucren un menor nivel de riesgo. La internacionalización necesita de mucho capital para poder consolidarse en el tiempo, es por esto por lo que se requiere la reinversión total de utilidades por un periodo extendido de años para que esta acción rinda sus frutos. Sin embargo, un factor que atenta en contra de esta realidad es el crecimiento demográfico familiar, ya que exige una porción importante de las utilidades.

También quebranta la voluntad de los socios de invertir sus ganancias en el proceso exportador, la existencia de expectativas negativas con relación a la evolución del entorno; este aspecto tiene una significación especial en el caso venezolano, ante la incertidumbre imperante en el plano político, jurídico, económico y social, que impactan en la reinversión de utilidades de los empresarios familiares exportadores zulianos, explicando los exiguos montos de exportación del sector. Lo anterior, exige que los empresarios comprendan que la base de cualquier proceso de internacionalización se edifica en la disposición y gestión de distintos tipos de recursos que la empresa posee (tecnológicos, organizacionales, humanos, financieros, entre otros) que le permiten construir una ventaja competitiva en el mercado internacional. Si se parte del hecho de que la empresa familiar se enfrenta a este escenario en condiciones desventajosas, es de esperar que al igual que en otros contextos estudiados, esta se retrase en iniciarlo y se conduzca de manera más lenta. A partir de la creación de una unidad administrativa para las actividades internacionales se dará más organización para llevar adelante un plan determinado para incrementar el porcentaje de producto exportado. Esta decisión permitirá alcanzar un crecimiento sostenido de tales actividades, pues este departamento contará con los recursos y capacidades necesarias para competir exitosamente en el mercado mundial.

Estas nuevas circunstancias permitirán a los negocios de familia crear condiciones para aumentar la oferta exportable con base a situaciones generadas por la empresa, que superen el patrón de exportaciones esporádicas prevalecientes hasta el momento. La exportación no puede ser algo para salvar una coyuntura, sino que tiene que ser un objetivo de mediano y largo plazo. Es por ello, que las motivaciones para internacionalizarse deben descansar sobre la base de una estrategia deliberada para la penetración de mercados externos y el compromiso de la gerencia con la actividad. Es necesario superar el antiguo esquema de explotación de las ventajas comparativas y comenzar a crear ventajas competitivas que surjan sobre la base de la utilización del cúmulo de recursos naturales aprovechables en el país y en la región.

El desarrollo de la competitividad requiere de estrategias que aseguren la eficiencia, para ello es necesario emprender múltiples esfuerzos que conduzcan a la creación de nuevos conocimientos y habilidades para sacarle provecho a los recursos que se tengan disponibles. Hallar la ventaja o ventajas competitivas, es un desafío que tiene que ir descubriendo la propia empresa familiar. Quizás una primera tarea para cumplir con tan retador objetivo es la de analizar las fortalezas y debilidades de la organización en la expansión de su mercado al exterior, pero también exigirá el examen de las oportunidades y amenazas que presenta un entorno internacional cada vez más turbulento y riesgoso.

\section{CONCLUSIONES}

De acuerdo al trabajo presentado y a los resultados obtenidos, se pueden plantear las siguientes conclusiones principales:

1.- Las empresas familiares estudiadas evidenciaron debilidades en su gestión estratégica de expansión internacional producto de la falta de recursos de tipo organizacional, tecnológico, financiero y humanos, entre otros.

2.- El modelo prospectivo propuesto, además de plantear una mayor inversión en tecnología y formación de recursos humanos para el comercio internacional, precisó la necesidad de afianzar los valores que impulsaron el espíritu empresarial, los cuales deben ser socializados y transferidos a los miembros de la siguiente generación de propietarios y sus empleados, siendo fundamental la puesta en práctica de órganos de gobierno que regulen el desempeño de los familiares en el negocio. 
3.- Finalmente, se subraya la importancia de este estudio al poner en contexto los retos y desafíos que asumen las empresas familiares en el ámbito internacional que, unido al análisis del espacio nacional exige la definición de reglas claras de la dinámica gubernamental en la esfera exportadora para garantizar la continuidad y supervivencia de las empresas familiares.

\section{REFERENCIAS}

Acosta de Mavárez, A., Molina-Quiroz, C., Andino-Chancay, T., y Rodríguez-López, V., Sistema familiar y continuidad de las empresas familiares, https://doi.org/10.31876/rcs.v25i4.30532, Revista de Ciencias Sociales, 25(4), 265-275 (2019)

Alayo, M., Maseda, A., Iturralde, T., y Arzubiaga, U., Internationalization and entrepreneurial orientation of family SMEs: The influence of the family carácter, https://doi.org/10.1016/j.ibusrev.2018.06.003, International Business Review, 28(1) (2019)

Augusto-Felício, J., Meidutè, L., y Kyvik, Ø., Global mindset, cultural context, and the internationalization of SMEs, https://doi.org/10.1016/j.jbusres.2016.04.054, Journal of Business Research, 69(11) 4924-4932 (2016)

Aznarez, J., y Vilaseca A., Estructura de financiamiento de la empresa familiar, Revista de Antiguos Alumnos del Instituto de Estudios Empresariales de Montevideo, 7(13), 18 (2004)

Berrone, P., Cruz C., y Gomez-Mejia, L., Socioemotional wealth in family firms: Theoretical dimensions, assessment approaches, and agenda for future research, https://doi.org/10.1177/0894486511435355, Family Business Review, 23(3), 258-279 (2012)

Boellis, A., Mariotti, S., Minichilli, A., y Piscitello, L., Family involvement and firms' establishment mode choice in foreign markets, https://doi.org/10.1057/jibs.2016.23, Journal of International Business Studies, 47(8), 929-950 (2016)

Buccieria, D., Javalgi, R., y Cavusgil, E., International new venture performance: Role of international entrepreneurial culture, ambidextrous innovation, and dynamic marketing capabilities, https://doi.org/10.1016/j.ibusrev.2020.101665, International Business Review, 29(2) (2020)

Cabrera-Suárez, M. K., Déniz-Déniz, M. D. L. C., y Martín-Santana, J. D., The setting of non-financial goals in the family firm: The influence of family climate and identification, https://doi.org/10.1016/j.jfbs.2014.05.003, Journal of Family Business Strategy, 5(3), 289-299 (2014)

Clark, D., Li, D., y Shepherd, D., Country familiarity in the initial stage of foreign market selection, https://doi.org/10.1057/s41267-017-0099-3, Journal of International Business Studies, 49, 442-472 (2018)

Cóndor, E., Bustamante, M., Lapo, M., y Campos, R., Factores Intrínsecos de motivación y liderazgo en una empresa familiar ecuatoriana de telecomunicaciones, http://dx.doi.org/10.4067/S0718-07642018000400205, Información Tecnológica, 29(4), 205-216 (2018)

Corral, S. L., y Rébori, A., La diversificación del negocio familiar en países emergentes, desde la perspectiva de la riqueza socioemocional. Revisión de la literatura, https://doi.org/10.25100/cdea.v35i63.6938, Cuadernos de Administración, 35(63), 89-100 (2019)

De la Hoz-Dominguez, E., Fontalvo-Herrera, T., y Escorcia-Guzman, A., Creación de perfiles empresariales para compañías exportadoras mediante aprendizaje no supervisado, http://dx.doi.org/10.4067/S0718-07642019000600193, Información Tecnológica, 30(6), 193-200 (2019)

Garza-Ramos, M., Medina-Quintero, y otros cuatro autores, Los valores familiares y la empresa familiar en el Nordeste de México, Cuadernos de Administración, ISSN 0120 3592, 24(42), 315-333 (2011)

Godet, M., De la anticipación a la acción, manual de prospectiva y estratégica. Una indisciplina intelectual. Editorial Bouxareu- Alfaomega-Marcombo, Madrid, España (1999)

Hernández-Fernández, L., y Portillo-Medina, R., Profesionalización empresarial en empresas familiares: Una construcción teórica, Educosta, Barranquilla, Colombia (2016)

Hernández-Fernández, L., Portillo-Medina, R., Romero-Borré, J., y Hernández-Chacín, A., Gobierno corporativo y profesionalización empresarial en hipermercados y supermercados familiares en Venezuela, Revista Venezolana de Gerencia, ISSN 1315 9984, 20(69), 116-135 (2015)

Hurmerinta, L., Paavilainen-Mäntymäki, E., y Hassett, M. E., Tempus fugit: A Hermeneutic Approach to the Internationalisation Process, http://dx.doi.org/10.1007/s11575-016-0303-x, MIR: Management International Review, 56 (6), 805-825 (2016)

Kampouri, K., Plakoyiannaki, E., y Leppäaho, T., Family business internationalization and networks: Emerging pathways, http://dx.doi.org/10.1108/JBIM-04-2015-0066, Journal of Business \& Industrial Marketing, 32(3) 357-370 (2017)

Kellermanns, F. W., Eddleston, K. A., y Zellweger, T. M., Extending the Socioemotional Wealth Perspective: A Look at the Dark Side, https://doi.org/10.1111/j.1540-6520.2012.00544.x, Entrepreneurship Theory and Practice, 36 (6), 1175$1182(2012)$

Liang, X., Wang, L., y Cui, Z., Chinese private firms and internationalization: Effects of family involvement in management and family ownership, https://doi.org/10.1177/0894486513480885, Family Business Review, 27(2), 126$141(2014)$ 
Marín-González, F., Cabas de J. L., Cabas, L., y Paredes-Chacín, A. J., Formación integral en profesionales de la ingeniería. Análisis en el plano de la calidad educativa, http://dx.doi.org/10.4067/S0718-50062018000100013, Formación Universitaria, 11(1), 13-24 (2018)

Martelo, R., Moncaris, L., y Velez, L., Integración del Ábaco de Régnier, Encuestas y lluvia de ideas en la definición de variables claves en estudios prospectivos, http://dx.doi.org/10.4067/S0718-07642016000500025, Información Tecnológica, 27(5), 243-250 (2016)

Metsola, J., Leppäaho, T., Paavilainen-Mäntymäki, E., y Plakoyiannaki, E., Family business internationalization process: state of the art and ways to follow, https://doi.org/10.1016/j.ibusrev.2020.101665, International Business Review, 29(2) (2020)

Niittymies, A., y Pajunen, K., Cognitive foundations of firm internationalization: A systematic review and agenda for future research, https://doi.org/10.1016/j.ibusrev.2019.101654, International Business Review, 29(4) (2020)

Petrů, N., Tomášková, A., y Krošláková, M., Diversification of export territories of family businesses as a tool of their further development, http://dx.doi.org/10.21511/ppm.17(3).2019.25, Problems and Perspectives in Management, 17(3), 306-322 (2019)

Pukall, T. J., y Calabrò, A., The internationalization of family firms: A critical review and integrative model, https://doi.org/10.1177/0894486513491423, Family Business Review, 27(2), 103-125 (2014)

Romero, J., Dinámica de la internacionalización de la empresa familiar. Tesis doctoral, Mimeo, Universidad del Zulia, Maracaibo, Venezuela (2009)

Romero, J., Hernández, L., y Bracho, N., Incidencia del entorno en las exportaciones de las empresas familiares del estado Zulia-Venezuela, Revista Opción, 31(77), 126-144 (2015)

Tápies, J., y Ward, J., Family values and value creation. The fostering of enduring values within family-owned businesses. Editor Palgrave Macmillan, Londres, Reino Unido (2008)

Tsang, E. W. K., Learning from overseas venturing experience: The case of Chinese family businesses, http://dx.doi.org/10.1016/S0883-9026(00)00052-5, Journal of Business Venturing, 17, 21-40 (2002) 
